\title{
Some Comments on Chern-Simons Gauge Theory
}

T. R. Ramadas ${ }^{1}$, I. M. Singer, and J. Weitsman

Department of Mathematics, M.I.T., Cambridge, MA 02139, USA

\begin{abstract}
Following M. F. Atiyah and R. Bott [AB] and E. Witten [W], we consider the space of flat connections on the trivial $S U(2)$ bundle over a surface $M$, modulo the space of gauge transformations. We describe on this quotient space a natural hermitian line-bundle with connection and prove that if the surface $M$ is now endowed with a complex structure, this line bundle is isomorphic to the determinant bundle. We show heuristically how path-integral quantisation of the Chern-Simons action yields holomorphic sections of this bundle.
\end{abstract}

\section{Introduction}

In [W], Witten studied a $2+1$ dimensional quantum Yang-Mills theory, with an action consisting purely of the Chern-Simons term,

$$
C S(\mathbf{A})=\frac{1}{4 \pi} \int \operatorname{Tr}\left(\mathbf{A} d \mathbf{A}+\frac{2}{3} \mathbf{A} \mathbf{A} \mathbf{A}\right) .
$$

He obtained the Jones polynomials of knots on $S^{3}$ and their extensions to other 3-manifolds as expectation values of Wilson loop functionals. A key point was the identification of the quantum state space as the space of holomorphic sections of a line bundle.

We first describe this line bundle from an algebraic point of view. Let $M$ denote a compact 2-manifold without boundary (with genus $\mathbf{g} \geqq 3$ - the other cases can be treated with analogous results), $\mathscr{A}$ the space of connections on the trivial $S U(2)$ bundle on $M, \mathscr{A}_{F}$ the space of flat connections, $\mathscr{A}_{F}^{s}$ the space of irreducible flat connections, and $\mathscr{G}$ the group of gauge transformations. Then it is well-known that $\mathscr{A}_{F}^{s} / \mathscr{G}$ is in a natural way a symplectic manifold. A choice of conformal structure $M_{c}$ on $M$ endows $\mathscr{A}_{F}^{s} / \mathscr{G}$ with a compatible Kähler structure, and it can be

${ }^{1}$ On leave from the School of Mathematics, Tata Institute of Fundamental Research, Homi Bhabha Road, Bombay 400005, India

I.M.S. and T.R.R. supported by DOE grant DE-FG02-88ER25066. J.W. supported by NSF Mathematical Sciences post-doctoral research scholarship 8807291 
identified with the moduli space $\mathscr{S}$ of stable vector bundles on $M_{c}$ of rank 2 and trivial determinant [NS, AB]. The Kähler structure on $\mathscr{S}$, suitably normalised, comes from an ample class on $\overline{\mathscr{S}}$, the moduli space of semistable vector bundles, (which in turn can be identified with the quotient $\mathscr{A}_{F} / \mathscr{G}$ ). In fact, by recent results of Narasimhan and Drezet [ND] there is a unique line bundle $\mathscr{L}$ in this class.

The state-space of relevance for the quantum field theory is the space of global sections $H^{0}\left(\overline{\mathscr{S}}, \mathscr{L}^{k}\right)$, where the integer $k$ multiplies the Chern-Simons action in functional integrals. Witten obtains this finite-dimensional space via holomorphic quantization of a classically constrained phase space. On physical grounds he concludes that the vector bundle $\mathscr{W}$ over Teichmuller space with fibre $H^{0}\left(\mathscr{S}, \mathscr{L}^{k}\right)$ is projectively flat, i.e., has a natural connection such that the curvature is a form with values in scalar endomorphisms. (Equivalently, parallel transport in the associated projective bundle has trivial holonomy.) Moreover, every 3-manifold $N$ such that $\partial N=M$ determines via the functional integral

\section{$\int \mathscr{D A} \exp i k C S(\mathbf{A})$}

a state vector $v_{N} \in H^{0}\left(\mathscr{S}, \mathscr{L}^{k}\right)$, and, given two such 3-manifolds $N_{1}$ and $N_{2}$ one has

$$
\left(v_{N_{1}}, v_{N_{2}}\right)=\int \mathscr{D} \mathbf{A} \exp i k C S(\mathbf{A}) \text {, }
$$

where the integral is over connections $\mathbf{A}$ on the 3-manifold $\tilde{N}$ obtained by identifying $N_{1}$ and $N_{2}$ along $M .^{2}$

This paper was motivated in part by trying to understand the existence of the projectively flat connection in terms of differential geometry. Some progress can be found in Sect. 2 where we prove

Theorem 1. There exists a natural hermitian line bundle $\mathscr{L}$ on $\mathscr{A}_{F} / \mathscr{G}$. Restricted to $\mathscr{A}_{F}^{s} / \mathscr{G}$, this line bundle carries a natural connection whose curvature is (up to a factor of $i)$ the standard symplectic form.

This theorem does not require a choice of conformal structure on $M$. However given a conformal structure, we can consider the determinant line bundle of the family of elliptic operators $\left\{\bar{\partial}_{\vec{A}} \mid \vec{A} \in \mathscr{A}_{F}^{s}\right\}$. If further a choice of compatible Riemannian metric on $M$ is made, the construction of Quillen [Q] yields a hermitian structure and connection on this bundle, and one can show that it descends to $\mathscr{S}$ as holomorphic hermitian line bundle $\mathscr{L}_{D}$ with connection. We then have

Theorem 2. $\mathscr{L}$ is isomorphic to the determinant bundle $\mathscr{L}_{D}$, as a hermitian line bundle with connection on $\mathscr{S}$.

Theorems 1 and 2 show that the fibres of the holomorphic bundle $\mathscr{W}$ imbed naturally into $C^{\infty}(\mathscr{S}, \mathscr{L})$.

In Sect. 3 we sketch a heuristic path-integral approach which yields the quantum state-space $H^{0}\left(\overline{\mathscr{S}}, \mathscr{L}^{k}\right)$ affirming Witten's geometric quantization. In Sect. 4 we point out some subtleties when the gauge group is $U(1)$ so that $\mathscr{A}_{F} / \mathscr{G}$ is the Jacobian. We conclude with some speculations concerning the correspondence $N \mapsto v_{N}$.

${ }^{2}$ The functional integrals can be defined only after a choice of metric on $\tilde{N}$. A suitably modified integral depending on a choice of framing of $\widetilde{N}$ turns out to only depend on the homotopy class of the framing. We will not consider the metric dependence in this paper 
Witten considered gauge groups $S U(n)$, with $n \geqq 2$. For simplicity we have limited our attention to the case $n=2$.

For a recent study of Chern-Simons gauge theory from the Schrödinger viewpoint see [DJT].

One of us (T.R.R.) would like to thank M.S. Narasimhan for very useful conversations.

\section{The Line Bundle}

2.1. As in the introduction, let $\mathscr{A}$ be the space of $s u(2)$-valued 1-forms on $M, \mathscr{A}_{F}$ the subspace of flat connections, $\mathscr{A}_{F}^{s}$ the submanifold of irreducible flat connections, $\mathscr{G}$ the space of gauge transformations. Also let $N$ be a 3-manifold with $\partial N=M$; in fact we assume given a diffeomorphism of a neighbourhood of $M$ in $N$ with $M \times[0,1)$.

Consider the following $U(1)$-valued function on $\mathscr{A} \times \mathscr{G}$ :

$$
\Theta(\vec{A}, g) \equiv \exp i\left(C S\left(\mathbf{A}^{\tilde{g}}\right)-C S(\mathbf{A})\right),
$$

where $\mathbf{A}$ and $\tilde{g}$ are extensions of $\vec{A}$ and $g$ into $N$ and $\mathbf{A}^{\tilde{g}}$ is the gauge transform of $\mathbf{A}$ by $\tilde{g}$. Such an extension of $g$ into $N$ is always possible in the case when the structure group is $S U(2)$ because $\pi_{1}(S U(2))=\pi_{2}(S U(2))=0$. We shall choose the extensions such that on $M \times[0,1), \mathbf{A}$ and $\tilde{g}$ are the pull-backs of $\vec{A}$ and $g$ respectively by the projection to $M$. Now $\Theta$ is independent of $N$ and the extensions $\mathbf{A}$ and $\tilde{g}$. In fact extensions $\left(\mathbf{A}_{1}, \tilde{g}_{1}\right)$ and $\left(\mathbf{A}_{2}, \tilde{g}_{2}\right)$ as above into $N_{1}$ and $N_{2}$ give a connection $\mathbf{B}$ and gauge transformation $h$ on $N=N_{1} \bigcup_{M}-N_{2}$ so that

$$
\begin{aligned}
\Theta\left(\mathbf{A}_{1}, \tilde{g}_{1}\right) \Theta\left(\mathbf{A}_{2}, \tilde{g}_{2}\right)^{-1} & =\exp i\left(C S\left(\mathbf{B}^{\tilde{h}}\right)-C S(\mathbf{B})\right) \\
& =1
\end{aligned}
$$

because $\operatorname{CS}\left(\mathbf{B}^{\tilde{h}}\right)-C S(\mathbf{B})$ is $2 \pi$ times an integer.

The function $\Theta$ is a cocycle:

$$
\Theta(\vec{A}, g) \Theta\left((\vec{A})^{g}, h\right)=\Theta(\vec{A}, g h)
$$

We define

$$
\mathscr{L} \equiv \mathscr{A}_{F}^{s} \times{ }_{\Theta} \mathbf{C},
$$

where on the right we mean the quotient by the equivalence relation:

$$
(\vec{A}, z) \sim\left(\vec{A}^{g}, \Theta(\vec{A}, g) z\right) .
$$

It is clear that $\mathscr{L}$ is a complex line bundle on $\mathscr{A}_{F}^{s} / \mathscr{G}$, and since $\Theta$ is $U(1)$-valued, it is a hermitian line bundle. One can also define the corresponding principal $U(1)$ bundle $\mathscr{U}$ in a similar fashion.

One can easily check that $\Theta$ is $C^{\infty}$ on $\mathscr{A} \times \mathscr{G}$ in the appropriate Sobolev norms. Integration by parts shows that its differential $d \Theta(\alpha, \phi)$ at $(\vec{A}, g)$ equals

$$
\frac{i}{4 \pi} \Theta \times\left\{\int_{M} \operatorname{Tr}\left(g^{-1} d g g^{-1} \alpha g\right)-\int_{M} \operatorname{Tr}\left(\vec{A}^{g} \wedge d_{\vec{A}^{g}} \phi\right)+2 \int_{M} \operatorname{Tr}\left(F_{\vec{A}^{g}} \phi\right)\right\},
$$

where $\alpha$ is a tangent vector to $\mathscr{A}$ [i.e., an $s u(2)$-valued 1 -form on $M$ ] and $\phi$ is an infinitesimal gauge transformation [i.e., an $s u(2)$-valued 0 -form]. 
We recall next the definition of the symplectic structure on $\mathscr{A}_{F}^{s} / \mathscr{G}$. On the space $\mathscr{A}$ of all connections, the two-form

$$
\widehat{\Omega}(\alpha, \beta) \equiv \frac{i}{2 \pi} \int_{M} \operatorname{Tr}(\alpha \wedge \beta)
$$

(where $\alpha$ and $\beta$ are tangent vectors at $A \in \mathscr{A}$ ) is closed. Restricted to $\mathscr{A}_{F}^{s}$ it is singular in the direction of gauge transformations:

$$
\int_{M} \operatorname{Tr}\left(d_{A} \phi \wedge \beta\right)=-\int_{M} \operatorname{Tr}\left(\phi \wedge d_{A} \beta\right)=0
$$

if $d_{A} \beta=0$ (the condition that $\beta$ be tangent to $\mathscr{A}_{F}^{s}$ ). In fact the restricted form descends to a form $\Omega$ on $\mathscr{A}_{F}^{s} / \mathscr{G}$ which is symplectic (modulo a factor of $i$ ) [AB].

Note that on $\mathscr{A}, \hat{\Omega}=d \hat{\omega}$, where the 1 -form $\hat{\omega}$ is defined by

$$
\hat{\omega}(\alpha)=\frac{i}{4 \pi} \int_{M} \operatorname{Tr}(\vec{A} \wedge \alpha) .
$$

We now check that $\hat{\omega}$, restricted to $\mathscr{A}_{F}^{s}$ is the pull-back [via the map $\left.\mathscr{A}_{F}^{s} \rightarrow \mathscr{A}_{F}^{s} \times 1 \hookrightarrow \mathscr{A}_{F}^{s} \times U(1) \rightarrow \mathscr{A} \times{ }_{\Theta} U(1) \rightarrow \mathscr{U}\right]$ of a unitary connection on $\mathscr{U}$ whose curvature is $\Omega$. First, $\hat{\omega}$ defines a connection one-form $\hat{\omega}_{P}$ on the principal $U(1)$ bundle $\mathscr{A}_{F} \times U(1)$. We have a twisted action of $\mathscr{G}$ on this bundle, and $\mathscr{U}$ is the quotient. Let $X$ be a vertical vector field for this action. Using the earlier formula for $d \Theta$ one verifies that $\hat{\omega}_{P}$ vanishes on $X$ and

$$
L_{X} \hat{\omega}_{P}=i_{X} d \hat{\omega}_{P}=i_{X} \hat{\Omega}=0,
$$

where $L_{X}$ is the Lie derivative. This completes the proof of Theorem 1.

Remarks. 1. In fact the above construction defines a continuous line bundle over $\mathscr{A} / \mathscr{G}$. One needs to check the following: if $g$ fixes $\vec{A} \in \mathscr{A}$, then $\Theta(\vec{A}, g)=1$. This is trivially true when $\vec{A}=0$ or when $g \equiv \pm$ Identity. When that is not the case and $g$ fixes $\vec{A}$, there exists $h \in \mathscr{G}$ such that

$$
\vec{A}^{h}=\left(\begin{array}{cc}
\vec{a} & 0 \\
0 & -\vec{a}
\end{array}\right),
$$

where $\vec{a}$ is a 1 -form, and

$$
h^{-1} g h=\left(\begin{array}{ll}
u & 0 \\
0 & \bar{u}
\end{array}\right),
$$

for some const $u$ with $|u|=1$. It is easy to check that $\Theta\left(\vec{A}^{h}, h^{-1} g h\right)=1$ and this implies $\Theta(\vec{A}, g)=1$.

2. The cocycle $\Theta$ was first considered, in a slightly different form, by Jackiw [J]. A construction similar to the above, of a line bundle over a loop group, (using the Wess-Zumino-Novikov-Witten term) appears in Mickelsson [M]. We thank one of the referees for pointing out the latter reference.

3 . It is worth pointing out the naturality of $\mathscr{L}$ : Introducing subscripts in an obvious way, a map of surfaces $\sigma: M_{1} \rightarrow M_{2}$ induces a map $\tilde{\sigma}: \mathscr{A}_{F, 2} / \mathscr{G}_{2} \rightarrow \mathscr{A}_{F, 1} / \mathscr{G}_{1}$ such that $\tilde{\sigma}^{*} \Omega_{1}=($ degree $\sigma) \Omega_{2}$. We also have a morphism of hermitian line bundles $\mathscr{L}_{2}^{\text {degree } \tilde{\sigma}} \rightarrow \mathscr{L}_{1}$ "over" $\tilde{\sigma}$ which preserves connections. 
To identify $\mathscr{L}$ with the determinant bundle we will need to know $\pi_{j}\left(\mathscr{A}_{F}^{s} / \mathscr{G}\right)$, $j=0,1,2$. We will use:

$$
\mathscr{A}_{F} / \mathscr{G} \equiv\left\{\text { conjugacy classes of representations of } \pi_{1}(M)\right\} \equiv \mathscr{R}\left(\pi_{1}\right) \text {. }
$$

Let $\left\{\left(a_{i}, b_{i}\right) \mid i=1, \ldots, \mathbf{g}\right\}$ be loops on $M$ which form a standard set of generators of $\pi_{1}(M)$ so that we have $\prod_{i} a_{i} b_{i} a_{i}^{-1} b_{i}^{-1}=1$. Then we can write $\mathscr{R}\left(\pi_{1}\right) \equiv Z_{\pi} / S U(2)$, where

$$
Z_{\pi}=\left\{A_{1}, \ldots, A_{\mathbf{g}}, B_{1}, \ldots, B_{\mathbf{g}} \mid A_{i}, B_{i} \in S U(2), \text { with } \prod_{i} A_{i} B_{i} A_{i}^{-1} B_{i}^{-1}=1\right\}
$$

and $S U(2)$ acts adjointly on $Z_{\pi}$. We will let $Z_{\pi}^{s} \subset Z_{\pi}$ denote the irreducible representations of $\pi_{1}(M)$. It is easy to see that $Z_{\pi}^{s}=Z_{\pi} \backslash Y$, where $Y$ is the orbit of $T \times \ldots \times T$ under the adjoint action of $S U(2), T$ being the standard diagonal $U(1)$ subgroup of $S U(2)$.

We now prove the

Lemma. $\mathscr{R}\left(\pi_{1}\right)$ is connected and simply connected; $\pi_{2}\left(\mathscr{R}\left(\pi_{1}\right)\right)=\mathbf{Z}$.

Proof. The projection $Z_{\pi}^{s} \rightarrow \mathscr{R}\left(\pi_{1}\right)$ is a locally trivial fibration with fibre $S O(3)$. We show below that $Z_{\pi}^{s}$ has $\pi_{0}=0, \pi_{1}=0$, and $\pi_{2}=\mathbf{Z}$. Hence the same result holds for $\mathscr{R}\left(\pi_{1}\right)$.

Let $Z$ denote the product of $2 \mathbf{g}$ copies of $S U(2)$ :

$$
Z \equiv\left\{A_{1}, \ldots, A_{\mathbf{g}}, B_{1}, \ldots, B_{\mathbf{g}} \mid A_{i}, B_{i} \in S U(2)\right\} .
$$

The set $Y$ is a submanifold in $Z$ of codimension $4 \mathbf{g}-2$; since $\mathbf{g}>2, Z \backslash Y$ satisfies $\pi_{0}=0, \pi_{1}=0$, and $\pi_{2}=0$. Consider the map $R:(Z \backslash Y) \rightarrow S U(2)$ given by

$$
R\left(\left(A_{i}, B_{i}\right)\right)=\prod_{i} A_{i} B_{i} A_{i}^{-1} B_{i}^{-1} .
$$

Because this (proper) map has a differential of maximal rank at every point, it is a fibration. The space $Z_{\pi}^{s}$ is the inverse image of the identity element under $R$, and the standard exact sequence in homotopy proves that $\pi_{0}\left(Z_{\pi}^{s}\right)=0, \pi_{1}\left(Z_{\pi}^{s}\right)=0$. To see that $\pi_{2}\left(Z_{\pi}^{s}\right)=\mathbf{Z}$ we observe that the map $\pi_{3}(Z \backslash Y) \rightarrow \pi_{3}(S U(2))$ induced by $R$ is zero.

2.2. Choose a conformal structure $c$ on $M$. The space $\mathscr{A}^{s} / \mathscr{G}$ can then be identified [NS] with the moduli space $\mathscr{S}$ of stable vector bundles of rank 2 and trivial determinant on $M_{c}$. In fact, by [S] $\mathscr{A}_{F} / \mathscr{G}$ is a complete complex algebraic variety - the moduli space $\overline{\mathscr{S}}$ of (s-equivalence classes of semistable vector bundles - in which $\mathscr{S}=\mathscr{A}^{s} / \mathscr{G}$ sits as the smooth part.

The complex structure on $\mathscr{A}^{s} / \mathscr{G}$ can also be obtained [AB] via the $\star$-operator on 1-forms defined by the conformal structure $c$. The form $\Omega$ is of type $(1,1)$ with respect to any one of these holomorphic structures; $\mathscr{L}$ has therefore a unique holomorphic structure such that the unitary connection $\omega$ is compatible with it.

We proceed to identify the line bundle constructed above with the determinant bundle $\mathscr{L}_{D}$ of the family of $\bar{\partial}$ operators parametrised by $\mathscr{S}$. We will first define $\mathscr{L}_{D}$ as a bundle on $\mathscr{S}$. In [Q] Quillen constructs a determinant line bundle $\tilde{\mathscr{L}}_{D}$ on $\mathscr{A}$. This bundle has a hermitian metric and connection invariant under a lift of the action of $\mathscr{G}$, and its curvature is $\widehat{\Omega}$. Restricted to $\mathscr{A}_{F}^{s}$ it descends to give $\mathscr{L}_{D}$. The 
curvatures of $\mathscr{L}_{D}$ and $\mathscr{L}$ coincide, and using the above lemma one sees that $\mathscr{L}_{D}$ and $\mathscr{L}$ are isomorphic.

We now give the details. First, it is enough to consider $\tilde{\mathscr{L}}_{D}$ on the neighbourhood $\mathscr{A}^{s}$ of $\mathscr{A}_{F}^{s}$ consisting of $\vec{A}$ 's which define stable bundles. On this set the bundle $\widetilde{\mathscr{L}}_{D}$ is easy to describe. We have $[\mathrm{NS}] \operatorname{ker} \bar{\partial}_{\vec{A}}=H^{0}\left(M_{c}, \bar{\partial}_{\vec{A}}\right)=0$ and so the vector spaces coker $\bar{\partial}_{\vec{A}}=H^{1}\left(M_{c}, \bar{\partial}_{\vec{A}}\right)$ form a holomorphic vector bundle over $\mathscr{A}^{s}$ of rank $2(\mathrm{~g}-1)$ and $\widetilde{\mathscr{L}}_{\boldsymbol{D}}$ is the determinant bundle of this vector bundle. We have, for $g \in \mathscr{G}$ the relation $\bar{\partial}_{(\vec{A})^{g}}=g^{-1} \circ \bar{\partial}_{\vec{A}} \circ g$ which shows that the action of $\mathscr{G}$ on $\mathscr{A}^{s}$ lifts to the bundle of cokernels. One can check, using the definition of holomorphic structure on this bundle, that the lifted action is by holomorphic isomorphisms. Thus the action of $\mathscr{G}$ on $\mathscr{A}^{s}$ lifts to an action on $\tilde{\mathscr{L}}_{D}$ by holomorphic transformations, which, further, preserves the Quillen metric in it. This implies that the action preserves the canonical connection on the bundle: denoting by $\tilde{g}$ the lift of $g \in \mathscr{G}$ we have

$$
\nabla_{X}\left((\tilde{g})^{-1} \circ \mu \circ g\right)=(\tilde{g})^{-1} \circ\left(\nabla_{g_{*}(X)} \mu\right) \circ g
$$

for any section $\mu$. Let us now restrict $\tilde{\mathscr{L}}_{D}$, as a hermitian line bundle with connection, to $\mathscr{A}_{F}^{S}$, continuing to denote it as $\tilde{\mathscr{L}}_{D}$. We now define $\mathscr{L}_{D}$ as a line bundle over $\mathscr{S}$ by taking the quotient of $\tilde{\mathscr{L}}_{D}$ by the action of $\mathscr{G}$. [The isotopy subgroup of $\mathscr{G}$ at any $\vec{A} \in \mathscr{A}_{F}^{s}$ is \pm Identity. Since $H^{1}\left(M_{c}, \bar{\partial}_{\vec{A}}\right)$ has even rank this subgroup acts trivially on $\tilde{\mathscr{L}}_{D}$ as well.]

We need to check that the connection descends. Let $\mu$ be any covariant section of $\tilde{\mathscr{L}}_{D}$ : i.e., $(\tilde{g})^{-1} \circ \mu \circ g=\mu, X$ a covariant vector field. The covariance of the connection shows that $\nabla_{X} \mu$ is covariant. We will now show that $\nabla_{X} \mu=0$ if $X$ is vertical. Let $\mathcal{O}$ be a $\mathscr{G}$ orbit in $\mathscr{A}_{F}^{\mathrm{s}}$. On $\mathcal{O}$ we can write $\nabla_{X} \mu=\kappa(X) \mu$, where $\kappa$ is a linear functional on $\{$ invariant vertical vector fields on $\mathcal{O}\}=$ Lie $\mathscr{G}$, the Lie algebra of $\mathscr{G}$. Since the curvature $\hat{\Omega}=0$ on $\mathcal{O}$, we have $\kappa\left(\left[X_{1}, X_{2}\right]\right)=0$. Since Lie $\mathscr{G}$ is perfect, $\kappa=0$.

From the lemma one concludes easily that $H^{2}(\mathscr{S}, \mathbf{Z})=\mathbf{Z}$. This, together with the fact that $\mathscr{S}$ is simply connected, implies that two hermitian line bundles with the same curvature 2-form are isomorphic as line bundles with connection, thus proving Theorem 2.

Remarks. 1. One can check that $H^{1}\left(M_{c}, \bar{\partial}_{\vec{A}}\right) \simeq H^{0}\left(M_{c}, K_{\chi}\right)^{*}$ when $\vec{A}$ is flat, $\chi$ is the associated representation of $\pi_{1}(M)$ in $S U(2)$ and $K_{\chi}$ the canonical line bundle twisted by $\chi$. The latter space has a natural inner product $\langle\tau, \xi\rangle=\int_{M}(\tau, \wedge \bar{\xi})$. Hence the line $L_{\chi} \equiv \Lambda^{2 \mathrm{~g}-2} H^{0}\left(M_{c}, K_{\chi}\right)$ has a natural inner product. The line bundle $\left\{L_{\chi}\right\}_{\chi \in Z_{\pi}^{s}}$ descends to $R\left(\pi_{1}\right)$ as a line bundle $\hat{\mathscr{L}}^{*}$ with hermitian structure. If one follows the path of defining a complex structure on $R\left(\pi_{1}\right)$ as in [NS], one could similarly define a holomorphic structure on $\hat{\mathscr{L}}^{*}$. We then have isomorphisms of holomorphic line bundles $\mathscr{L}=\mathscr{L}_{D}=\hat{\mathscr{L}}$.

However the Quillen metric on $\mathscr{L}_{D}$ differs from the metric on $\hat{\mathscr{L}}$ described above by the function $\operatorname{det} \Delta_{\chi}$, where $\Delta_{\chi}$ is the Laplacian on functions equivariant under $\chi$.

2. The determinant bundle is an algebraic object, and in fact exists over $\overline{\mathscr{S}}$. In fact since Pic $\overline{\mathscr{S}} \sim \operatorname{Pic} \mathscr{S} \sim \mathbf{Z}[\mathrm{ND}]$ every holomorphic line bundle on $\mathscr{S}$ extends uniquely as an invertible sheaf on $\overline{\mathscr{S}}$. 


\section{Holomorphic Quantisation via Formal Path-Integrals}

Consider again the Chern-Simons action functional

$$
C S(\mathbf{A})=\frac{1}{4 \pi} \int \operatorname{Tr}\left(\mathbf{A} d \mathbf{A}+\frac{2}{3} \mathbf{A A A}\right),
$$

where $\mathbf{A}$ is a connection on the trivial $S U(2)$ bundle on a 3-manifold. When the 3-manifold is diffeomorphic to $M \times[0,1]$, with $M$ a 2-manifold, we can write

$$
\mathbf{A}=A(t)+A_{0}(t) d t
$$

where $A(t)$ and $A_{0}(t)$ are a one parameter family of $s u(2)$-valued one- and zeroforms respectively on $M$ parametrised by $t \in[0,1]$. The Chern-Simons action becomes then:

$$
C S\left(A, A_{0}\right)=\frac{1}{4 \pi} \int d t \int_{M} \operatorname{Tr}\left(2 A_{0} F_{A}-A \frac{d A}{d t}\right) .
$$

Standard constraint analysis à la Dirac leads to $\mathscr{A}_{F}^{\mathbb{s}} / \mathscr{G}$, with its symplectic structure, as the reduced phase space. A conformal structure $c$ on $M$ determines (via the $\star$ operator) a compatible complex structure and Kähler metric on $\mathscr{A}^{s} / \mathscr{G}$ and this holomorphic polarisation determines (for $k=1$ ) the state-space of [W], described in Sect. 1.

Our purpose in this section is to obtain the state-space $H^{0}\left(\overline{\mathscr{S}}, \mathscr{L}^{k}\right)$ via the functional integral directly. We emphasize that the arguments in this section are not rigorous. Some of them are standard, but the one which suggests the holomorphy of the sections of $\mathscr{L}$ obtained by functional integrals is, to our knowledge, new.

Consider therefore the functional integral

$$
\Phi\left(\vec{A}, A_{(0, M)}\right)=\int_{\mathbf{A}(0)=\vec{A}+A_{(0, M)} d t} \mathscr{D}_{N} \mathbf{A} \exp i k C S(\mathbf{A}) \prod_{C} W_{\mathbf{A}}(C),
$$

where $\vec{A}$ is an $s u(2)$-valued 1 -form on $M, A_{(0, M)}$ a $s u(2)$-valued 0 -form, $N$ is a 3-manifold such that $\partial N=M$, the subscript on $\mathscr{D}$ refers to the fact that the functional integral is over $\mathbf{A}$ on $N$ and $\prod_{C} W_{\mathbf{A}}(C)$ is a product of Wilson loops in $N$ not intersecting $M$. We will argue that $\Phi$ can be interpreted as a holomorphic section of $\mathscr{L}^{k}$.

Choose a neighbourhood (not intersecting any of the loops $C$ ) of $M$ in $N$ diffeomorphic to $M \times[0,1)$ and in fact fix such a diffeomorphism, letting $t$ denote as before the co-ordinate along $[0,1)$. The functional integral $\Phi$ above can be expressed as

$$
\begin{aligned}
\Phi\left(\vec{A}, A_{(0, M)}\right)= & \int_{\mathbf{A}(0)=\vec{A}+A_{(0, M)} d t} \mathscr{D}_{N} \mathbf{A} \exp i k C S(\mathbf{A}) \prod_{C} W_{\mathbf{A}}(C) \\
= & \int_{A(0)=\vec{A}, A_{0}(0)=A_{(0, M)}} \mathscr{D} A(t) \mathscr{D} A_{0}(t) \exp i k C S\left(A, A_{0}\right) \\
& \times \int_{\mathbf{A}(1)=A(1)+A_{0}(1) d t} \mathscr{D} \backslash M \times[0,1) \\
= & \mathbf{A} \exp i k C S(\mathbf{A}) \prod_{C} W_{\mathbf{A}}(C) \\
= & \int_{A(0)=\vec{A}, A_{0}(0)=A_{(0, M)}} \mathscr{D} A(t) \mathscr{D} A_{0}(t) \exp i k C S\left(A, A_{0}\right) \Psi\left(A(1), A_{0}(1)\right),
\end{aligned}
$$


where the effects of the integral over the fields on the rest of the manifold are subsumed in the functional $\Psi$. We will see below that the functional $\Phi$ is independent of $A_{(0, M)}$ and $\Psi$ of $A_{0}(1)$, so we can write

$$
\Phi(\vec{A})=\int_{(A(0)=\vec{A})} \mathscr{D} A(t) \mathscr{D} A_{0}(t) \exp i k C S\left(A, A_{0}\right) \Psi(A(1)) .
$$

The expression

$$
C S\left(A, A_{0}\right)=\frac{1}{4 \pi} \int d t \int_{M} \operatorname{Tr}\left(2 A_{0} F_{A}-A \frac{d A}{d t}\right)
$$

shows that the above functional integral is nonzero only for paths $A(t)$ in $\mathscr{A}_{F}$. More precisely,

$$
\int \mathscr{D} A_{0}(t) \exp \frac{i k}{4 \pi} \int_{M} \operatorname{Tr}\left(2 A_{0} F_{A}\right)=\frac{\delta\left(F_{A}(t)=0\right)}{2 k \mathscr{D}_{1}(t)}
$$

where $\mathscr{D}_{1}(t)=\sqrt{\operatorname{det} L(t)^{*} L(t)}$, and $L(t)=\left.d_{A(t)}\right|_{\operatorname{ker} d_{A(t)}^{*}}$ is the differential (in the normal direction to $\left\{F_{A}(t)=0\right\}$ ) of the map $A \mapsto F_{A}$ of 1 -forms to 2 -forms. We have our first result: $\Phi$ is supported on connections $\vec{A}$ which are flat, i.e., $\Phi(\vec{A})=0$ unless $F_{\vec{A}}=0$.

We next demonstrate that the functional $\Phi$ is a section of $\mathscr{L}^{k}$, i.e., $\vec{A} \mapsto(\vec{A}, \Phi(\vec{A}))$ is a section of $\mathscr{L}^{k}$. Because of the equivalence relation defining $\mathscr{L}$, we need to check $\Phi\left(\vec{A}^{g}\right)=\Theta(\vec{A}, g)^{k} \Phi(\vec{A})$. Since $\mathscr{G}$ is connected, it suffices to verify this at the Lie algebra level: if $\phi$ is an infinitesimal gauge transformation,

$$
d /\left.d t\right|_{t=0} \Phi\left(\vec{A}^{e^{t \phi}}\right)=d /\left.d t\right|_{t=0} \Theta\left(\vec{A}, e^{t \phi}\right)^{k} \Phi(\vec{A})=\frac{i k}{4 \pi}\left(\int_{M} \operatorname{Tr}\left(\vec{A} \wedge d_{\vec{A}} \phi\right)\right) \Phi(\vec{A}),
$$

where in the second equation we have used the expression for the differential of $\Theta$ from Sect. 2.

Extend the function $\phi$ into $N$ so that it is supported within $M \times[0, \delta)$ and denote the extension by $\tilde{\phi}$. We have

$$
\Phi\left(\vec{A}^{t \phi}\right)=\int_{\left.\mathbf{A}(0)\right|_{M}=\vec{A}^{e^{t \phi}}} \mathscr{D}_{N} \mathbf{A} \exp i k C S(\mathbf{A}) \prod_{C} W_{\mathbf{A}}(C) .
$$

Make a change of variables $\mathbf{A} \mapsto \mathbf{A}^{e^{t \tilde{\phi}}}$. Assuming the integral is gauge invariant, we conclude because the Wilson loop functionals are gauge invariant that

$$
\Phi\left(\vec{A}^{e^{t \phi}}\right)=\int_{\left.\mathbf{A}(0)\right|_{M}=\vec{A}} \mathscr{D}_{N} \mathbf{A} \exp i k C S\left(\mathbf{A}^{e^{t \tilde{\phi}}}\right) \prod_{C} W_{\mathbf{A}}(C) .
$$

We note now that

$$
\left.\frac{d}{d t}\right|_{t=0} \operatorname{CS}\left(\mathbf{A}^{e^{t \tilde{\phi}}}\right)=\frac{1}{4 \pi} \int_{M} \operatorname{Tr}\left(\vec{A} \wedge d_{\vec{A}} \phi\right) \operatorname{CS}(\mathbf{A}),
$$

which proves the required covariance. (Note that the above argument also shows that $\Phi$ is independent of $A_{(0, M)}$, since we can change $A_{(0, M)}$ arbitrarily by a gaugetransformation that is the identity on the boundary.)

We now give a heuristic argument to show that $\Phi$ is a holomorphic section of $\mathscr{L}^{k}$. It pretends that $\overline{\mathscr{S}}$ is a smooth Kähler manifold or that $\mathscr{S}$ is compact. Of 
course the holomorphic structure comes from a choice of metric on $N$ needed to define the path integral - the metric on $N$ induces a metric in $M$ and hence a complex structure.

We will use the formal path integral expression for the heat kernel [FH] $e^{-T \Delta_{\omega_{k}}}$, where $\omega_{k}$ is the connection on $\mathscr{L}^{k}$ and $\Delta_{\omega_{k}}=d_{\omega_{k}}^{*} d_{\omega_{k}}$. If $\psi$ is a section of $\mathscr{L}^{k}$ we have

$$
e^{-T \Delta_{\omega_{k}} \psi(x)=} \int_{x:[0, T] \rightarrow \mathscr{S}, x(0)=x} \mathscr{D} x(t) \exp \left(-\int_{0}^{T} d t|d x / d t|^{2}-\int_{x(t)} \omega_{k}\right) \psi(x(T)),
$$

and $\exp \int \omega_{k}$ is the parallel transport operator from the fibre at $x(0)$ to the fibre at $x(T)$.

When $T \rightarrow \infty, e^{T \lambda_{0}} e^{-T \Delta_{\omega_{k}}}$ projects $\psi$ onto the eigenspace corresponding to the smallest eigenvalue $\lambda_{0}$. In our case we are working on a Kähler manifold and the curvature of the line bundle is a constant multiple of the Kähler form; hence $\Delta_{\omega_{k}}$ $=2 \bar{\partial}_{\omega_{k}}^{*} \bar{\partial}_{\omega_{k}}+k \times(\operatorname{dim} \mathscr{S})$ so that the above eigenspace is the space of holomorphic sections. That is,

$$
\lim _{T \rightarrow \infty} e^{T \lambda_{0}} \int_{x:[0, T] \rightarrow \mathscr{S}, x(0)=x} \mathscr{D} x(t) \exp \left(-\int_{0}^{T} d t|d x / d t|^{2}-\int_{x(t)} \omega_{k}\right) \psi(x(T))
$$

is a holomorphic section. We will now regulate our path integral to be exactly this.

Let us rewrite the expression for $\Phi$, taking into account our observation that only paths in $\mathscr{A}_{F}$ contribute:

$$
\begin{aligned}
\Phi(\vec{A}) & =\int_{A(0)=\vec{A}, A(t) \in \mathscr{A}_{F}} \mathscr{D} A(t) \prod_{t} \mathscr{D}_{1}(t) \exp \left(-\frac{i k}{4 \pi} \int d t \operatorname{Tr}\left(A \frac{d A}{d t}\right)\right) \Psi(A(1)) \\
& =\int_{A(0)=\vec{A}, A(t) \in \mathscr{A}_{F}} \mathscr{D} A(t) \prod_{t} \mathscr{D}_{1}(t) \exp \left(-k \iint_{A(t)} \hat{\omega}\right) \Psi(A(1)) .
\end{aligned}
$$

Note that now $A_{0}$ is out of the picture and yet the above functional integral has the gauge freedom $A(t) \mapsto g^{-1}(t) A(t) g(t)+g^{-t} d g(t)$. We now argue that the above integral descends to an integral over the space of paths on $\mathscr{S}$,

$$
\phi(x)=\int_{x:[0,1] \rightarrow \mathscr{S}, x(0)=x} \mathscr{D} x(t) \exp \left(-\int_{x(t)} \omega_{k}\right) \psi(x(1)),
$$

where $\phi$ and $\psi$ are sections of $\mathscr{L}$ determined respectively by $\Phi$ and $\Psi$ and $\omega$ is the connection one-form - the only point to note being that the volume of $\mathscr{G}$ cancels the determinant factor:

$$
\sqrt{\operatorname{det}\left(d_{A(t)}^{*} d_{A(t)}\right)_{0}}=\sqrt{\operatorname{det}\left(d_{A(t)}^{*} d_{A(t)}\right)_{1}}=\mathscr{D}_{1}(t) .
$$

(The operator $\star d_{A}$ gives an isomorphism between the eigenvectors with nonzero eigenvalue.)

The last functional integral can be regulated by adding a kinetic energy term to the action:

$$
\phi_{T}(x)=\int_{x:[0,1] \rightarrow \mathscr{S}, x(0)=x} \mathscr{D} x(t) \exp \left(-\frac{1}{T} \int_{0}^{1} d t|d x / d t|^{2}-\int_{x(t)} \omega_{k}\right) \psi(x(1)) .
$$


If we now make a change of variables $s=t T$ we get

$$
\phi_{T}(x)=\int_{x:[0, T] \rightarrow \mathscr{S}, x(0)=x} \mathscr{D} x(s) \exp \left(-\int_{0}^{T} d s|d x / d s|^{2}-\int_{x(s)} \omega_{k}\right) \psi(x(T)) .
$$

As $T \rightarrow \infty$, we get the projection of $\psi$ on the subspace of holomorphic sections, provided we renormalise by multiplying by $e^{T \lambda_{0}}$.

\section{The $U(1)$ Case; Concluding Remarks}

4.1. We consider the case of $U(1)$ bundles; two subtleties become more evident from our point of view.

First, the space $\mathscr{G}$ of maps from $M$ to $G$ is connected in the case when $G=S U(2)$, but when $G=U(1)$ the group of connected component of $\mathscr{G}$ is precisely $H^{1}(M, \mathbf{Z})$. The argument in 2.1 that the functionals $\Phi$ descend to sections of a line bundle on $\mathscr{A}_{F} / \mathscr{G}$ fails when $S U(2)$ is replaced by $U(1)$ because the gauge transformation $g$ need not extend into $M$. Also, there is no à priori reason now for the number $k$ to be an integer since the Chern-Simons action (on a manifold without boundary) is singlevalued modulo all gauge transformations. Let us, nevertheless see if the procedure does define a hermitian line bundle $\mathscr{L}$ with connection on $\mathscr{A}_{F} / \mathscr{G}$ $=H^{1}(M, \mathbf{R}) / H^{1}(M, \mathbf{Z})$ [where we have identified $\mathscr{A}_{F}$ with $\left.H^{1}(M, \mathbf{R})\right]$ by sending $\vec{A}$ to the class of $\frac{1}{2 \pi i} \vec{A}$. To define the factor of automorphy for a given gauge transformation $g$ one must now choose a three manifold $N$ such that $g$ extends into $N$. We can now evaluate $\Theta$ (we include the factor $k$ in the definition)

$$
\Theta_{k}(\vec{A}, g)=\exp \left(\frac{i k}{4 \pi} \int_{M}\left(g^{-1} d g \wedge \vec{A}\right)\right) .
$$

We see that for this to be a factor of au tomorphy for all gauge transformations [i.e., $\left.\Theta_{k}(\vec{A}, g h)=\Theta_{k}(\vec{A}, g) \Theta_{k}\left((\vec{A})^{g}, h\right)\right] k$ must be an even integer. We shall assume this to be the case. We shall denote the line bundle defined by taking $k=2$ by $l$.

Choosing a complex structure on $M$ yields one on $H^{1}(M, \mathbf{R})$; we can then identify $\mathscr{A}_{F} / \mathscr{G}=H^{1}(M, \mathbf{R}) / H^{1}(M, Z)$ with the Jacobian $J$.

Note that even if $k$ is an integer, different three-manifolds $N$ define vectors in different infinite-dimensional vector spaces, namely, sections of $l^{k / 2}$ on the (noncompact) space $H^{1}(M, \mathbf{R}) / \operatorname{Im} H^{1}(N, \mathbf{Z})$ with no further automorphic property to ensure that they descend to $J$; the obvious guess that these are all $\theta$-functions on the Jacobian may not be correct. [By $\operatorname{Im} H^{1}(N, \mathbf{Z})$ we mean the image of $H^{1}(N, \mathbf{Z})$ in $H^{1}(M, \mathbf{Z})$. We have the projection $H^{1}(M, \mathbf{R}) / \operatorname{Im} H^{1}(N, \mathbf{Z}) \rightarrow H^{1}(M, \mathbf{R}) / H^{1}(M, \mathbf{Z})$ and can pull back $l^{k / 2}$ by this map.]

Since $\pi_{1}(J) \neq 0$ it is interesting that path integral considerations have enabled us to choose a particular line bundle with connection whose curvature is the symplectic form out of a whole family of such bundles. Conventional geometric quantization does not do this.

4.2. When $N$ is a handle-body, it determines [A] a Lagrangian manifold $\mathscr{E}_{N}$ of $\mathscr{A}_{F}^{s} / \mathscr{G}$. The line bundle $\mathscr{L}$ is flat along $\mathscr{E}_{N}$; we will show that $\left.\mathscr{L}\right|_{\mathscr{E}_{N}}$ has a nonzero flat section. 
Let $\left\{\left(a_{i}, b_{i}\right) \mid i=1, \ldots, \mathbf{g}\right\}$ be loops on $M$ which form a standard set of generators of $\pi_{1}(M)$ so that we have $\prod_{i} a_{i} b_{i} a_{i}^{-1} b_{i}^{-1}=1$. Let $N$ be such that under the inclusion of fundamental groups $\pi_{1}(M) \rightarrow \pi_{1}(N)$ the $a_{i}$ are annihilated. The subspace $\mathscr{E}_{N}$ of $\mathscr{A}_{F} / \mathscr{G}$ corresponding to connections which have flat extensions into the interior of $N$ is a Lagrangian submanifold, i.e., a maximal submanifold on which $\Omega$ vanishes [A]. We give the brief argument: First, given two vectors $\alpha, \beta$ tangent to $\mathscr{E}_{N}$ at a point $A$,

$$
\begin{aligned}
\int_{M} \operatorname{Tr}(\alpha \wedge \beta) & =\int_{N} d \operatorname{Tr}(\alpha \wedge \beta) \\
& =\int_{N}\left(\operatorname{Tr}\left(d_{\mathbf{A}} \alpha \wedge \beta\right)-\operatorname{Tr}\left(\alpha \wedge d_{\mathbf{A}} \beta\right)\right) \\
& =0,
\end{aligned}
$$

where in the second and third line we have extended $A$ to a flat connection $\mathbf{A}$ and extended $\alpha, \beta$ to one-forms satisfying $d_{\boldsymbol{A}}(\cdot)=0$. This shows that $\mathscr{E}_{N}$ is isotropic. We next show that $\operatorname{dim} \mathscr{E}_{N}=\frac{1}{2} \operatorname{dim}\left(\mathscr{A}_{F} / \mathscr{G}\right)$. To see this, note that $\mathscr{\mathscr { E }}_{N_{\text {diffeo }}}=X / S U(2)$, where

$$
\underbrace{X=S U(2) \times \ldots \times S U(2)}_{\mathrm{g} \text { times }} \backslash S U(2)\{T \times \ldots \times T\} S U(2)^{-1} .
$$

$T$ is a maximal torus of $S U(2)$ and $S U(2)$ acts adjointly on $X$.

One can now also check that $\mathscr{E}$ is simply connected, and this implies that there is a nonzero flat section of of $\mathscr{L} \mid \mathscr{E}$, unique up to a nonzero scalar.

In fact this flat section exists even when the gauge group is $U(1)$. In that case we have to consider the bundle $l$, defined on $J \equiv \frac{H^{1}(M, \mathbf{R})}{H^{1}(M, \mathbf{Z})}$ by the factor of automorphy,

$$
\Theta_{1}(x, u)=\exp \left(-2 \pi i \int x \wedge u\right) .
$$

The one-form

$$
\hat{\omega}(x)[y] \equiv-\left(2 \pi i \int x \wedge y\right)
$$

defines a hermitian connection $\omega$ on this bundle. The curvature is

$$
\Omega[x, y]=-4 \pi i \int x \wedge y .
$$

Let $W$ be a subgroup of $H^{1}(M, \mathbf{Z})$ such that the subspace $\mathbf{R} W$ generated by it is of dimension $\mathbf{g}$ and isotropic for the intersection form. Then the expression for $\hat{\omega}$ shows: the image of $\mathbf{R} W$ in $J$ is a Lagrangian submanifold for $\Omega$, not simply connected, such that however the holonomy of $\omega$ is trivial on it.

4.3. One would expect a close relationship between a nonzero flat section (say $s_{N}$ ), along $\mathscr{E}_{N}$ and $v_{N}$. Computations in genus 1 show that $s_{N}$ does not extend to a holomorphic section of $\mathscr{L}$ (although if it did, such an extension would be unique). A saddle-point approximation to the functional integral shows that the sections $v_{N}$ are concentrated along $\mathscr{E}_{N}$, and this is sharper as $k$ increases. Again, computations 
in genus 1 show that $v_{N}$ is not the projection of $s_{N}$ (thought of as a distribution with support along $\left.\mathscr{E}_{N}\right)$ onto $H^{0}\left(\overline{\mathscr{S}}, \mathscr{L}^{k}\right)$. The relationship, if any, between $s_{N}$ and $v_{N}$ is subtler than the above.

\section{References}

[A] Atiyah, M.F.: New invariants of three and four manifolds. Proc. Symp. Pure Math. (AMS) 48 (1988)

[AB] Atiyah, M.F., Bott, R.: The Yang-Mills equation over Riemann surfaces. Phil. Trans. R. Soc. London A 308, 523-617 (1982)

[DJT] Dunne, G.V., Jackiw, R., Trugenberger, C.A.: Chern-Simons theory in the Schrödinger representation. MIT preprint MIT-CTP 1711 Ann. Phys. (to appear)

[FH] Feynman, R.P., Hibbs, A.R.: Quantum mechanics and path integrals. New York: McGraw-Hill 1965

[J] Jackiw, R.: Chern-Simons terms and cocycles in physics and mathematics. In Quantum field theory and quantum statistics. Batalin, I., Isham, C., Vikovisky, C. (eds.). Bristol, U.K.: A. Hilger 1987

[Q] Quillen, D.: Determinants of Cauchy-Riemann operators over a Riemann surface. Funct. Anal. Appl. 19, 31-34 (1986)

[M] Mickelsson, J.: String quantisation on group manifolds and the holomorphic geometry of Diff $S^{1} / S^{1}$. Commun. Math. Phys. 112, 653-662 (1987)

[NS] Narasimhan, M.S., Seshadri, C.S.: Stable and unitary vector on a compact surface. Ann. Math. 82, 540-567 (1965)

[ND] Narasimhan, M.S., Drezet, J.M.: TiFR preprint, 1988

[S] Seshadri, C.S.: Space of unitary vector bundles on a compact Riemann surface. Ann. Math. 85, 303-336 (1967)

[W] Witten, E.: Quantum field theory and the Jones polynomial. Institute for Advanced Study preprint IASSNS-HEP-88/33 (August 1988)

Communicated by A. Jaffe

Received March 30, 1989; in revised form May 19, 1989 